\title{
The Cisplatin/Serum Albumin System: a Reappraisal
}

\author{
Lara Massai $^{\mathrm{a}}$, Alessandro Pratesi $\mathrm{a}^{\mathrm{a}^{*}}$, Jurgen Gailer $^{\mathrm{b}}$, Tiziano Marzo ${ }^{\mathrm{c} \ddagger}$, Luigi Messori ${ }^{\mathrm{a}^{*} \ddagger}$ \\ aLaboratory of Metals in Medicine (MetMed), Department of Chemistry "U. Schiff”, University of Florence, \\ via della Lastruccia 3, 50019 Sesto Fiorentino, Italy. E-mail: luigi.messori@unifi.it \\ alessandro.pratesi@unifi.it
}

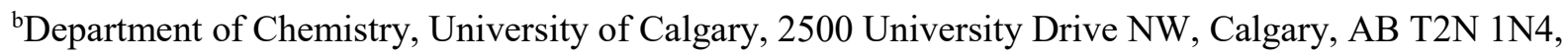
Canada

${ }^{\mathrm{c}}$ Department of Pharmacy, University of Pisa, via Bonanno Pisano 6, 56126, Pisa, Italy

$\$$ These authors contributed equally.

\begin{abstract}
Since the first approval of cisplatin for cancer treatment in 1978, a lot of attempts have been carried out to characterize in detail its interactions with serum albumin, by far the most important and most abundant plasma protein. The state of the art of those studies was recapitulated by Keppler and coworkers in an extensive review article which appeared in Chem. Rev. in 2006. Yet, the general picture was still rather incomplete at that time due to the lack of crystallographic data. Here, we report on the main achievements obtained on this system in the period 2006-2018 and try to describe what is now clearly ascertained and what are the still open issues. Remarkably, a detailed structural characterization of this metallodrug/protein system was recently obtained thanks to the resolution of the crystal structure of a cisplatin/serum albumin adduct; crystallographic results were nicely complemented by independent MS studies. In turn, metallomics investigations permitted to monitor platination of this serum protein in real blood samples. Thus, a rather complete description of the system was gained. In addition, the biological profile of cisplatin/serum albumin adducts was drafted in a specific study and its pharmacological implications discussed. The relevance and the impact of these novel results are herein critically analyzed.
\end{abstract}

Keywords: Cisplatin, Human Serum Albumin, crystal structure, metalation sites, mass spectrometry 


\section{Introduction}

Today, forty years after its first approval, cisplatin remains one of most important anticancer drugs in clinical use. Indeed, cisplatin (CDDP) and its analogues are currently included in about 50\% of medical regimens for cancer chemotherapy; cisplatin shows a pronounced efficacy most notably in testicular, head-and-neck and ovarian cancer treatment. It is generally accepted that CDDP exerts its antitumor effects mainly by DNA adduct formation, thereby perturbing DNA functions eventually leading to apoptosis. However, it is known that only $1-10 \%$ of cisplatin accumulated in the cell is able to reach nuclear DNA [1]. Probably, this fact is causally linked to the severe side effects seen in CDDP treated patients and, in particular, with the insurgence of neurotoxicity, ototoxicity and nephrotoxicity, as the remaining $90 \%$ of cisplatin is able to produce systemic toxic effects. In this view, the serum proteins, and in particular serum albumin, have attracted an increasing interest as "alternative targets" to sequester cisplatin [2]. Cisplatin is indeed a very reactive molecule capable of interacting tightly with several biomolecules of either high or low molecular weight; recently researchers' attention has moved to consider and characterize in depth the interactions of this drug with a variety of proteins from a structural and a molecular point of view [3].

Serum albumin is the most abundant protein in the blood plasma with a concentration in human serum about $0.6 \mathrm{mM}$ [4]. Human serum albumin (HSA) has a molecular mass of 66,438 Da and consists of three homologous domains, I, II, and III (Figure 1) [4-6]. Each domain contains subdomains A and $\mathrm{B}$ that show common structural motifs. The two principal regions responsible for ligand-binding to HSA are denoted as Sudlow's Sites I and II, located in subdomain IIA and IIIA (Figure 1), respectively $[4,7]$.

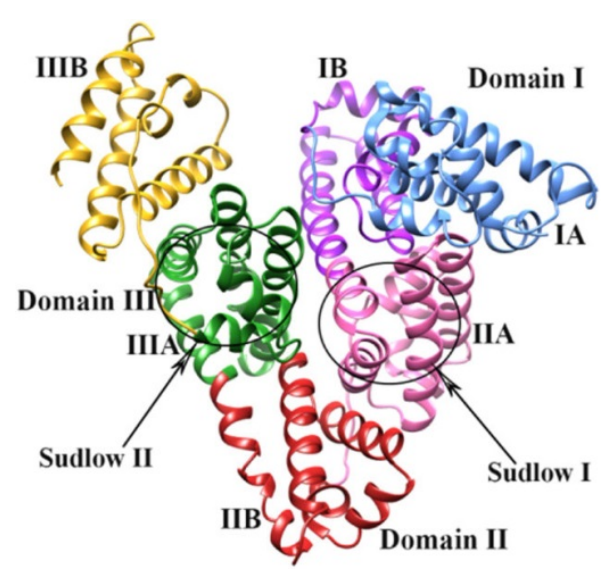

Figure 1. Structure of the Human Serum Albumin (HSA) with its domains, subdomains and Sudlow's binding sites I and II. Picture from Spectrochim Acta A Mol. Biomol. Spectrosc., 2017,183, 90-102. Albumin has an approximate half-life of 19 days and is primarily produced in the human liver with an average production of about $\sim 14 \mathrm{~g}$ of HSA per day [4]. Albumin plays a number of physiological 
roles and is responsible for $80 \%$ of the colloidal osmotic pressure of blood [4]. Remarkably, albumin is able to bind various endogenous molecules, including long-chain fatty acids, steroids, L-tryptophan, etc. $[4,8,9]$. Moreover, albumin is also involved in transporting various metal ions in the blood stream, including copper, zinc, calcium, etc. [4]. In addition, this vital protein is able to bind very many exogenous compounds and drugs, such as warfarin, ibuprofen, chlorpromazine and naproxen, with the affinity of their binding significantly affecting activity and half-life $[4,8,9]$; additionally it is the main carrier for a variety of metallodrugs.

Owing to the very high plasma concentration of serum albumin it may be easily predicted that a large fraction of injected cisplatin will bind/interact with this protein as supported by several studies and observations; yet, it is not clear whether the platinum fraction bound to serum albumin does conserve any significant pharmacological activity and/or may serve as a drug reservoir [10]. For the above reasons, the interactions of cisplatin with serum albumin have been repeatedly investigated, even at the molecular level.

An important study by the group of Peter Sadler appeared in 1998 [11], provided valuable information on the main aspects of those interactions. It was estimated that at least five platinum atoms could bind one albumin molecule through formation of coordinative bonds at a variety of protein side chains, mainly methionines and histidines.

The state of the art of the knowledge on the cisplatin/serum albumin system was later summarized in a review article by B. P. Espósito in 2002 [12] and by an extensive review by Keppler and coworkers in 2006 [2].

At that time the understanding of the cisplatin albumin system was roughly the following.

There was clear evidence that cisplatin in vitro might bind tightly serum albumin forming stable 1:1 adducts. Under physiologically relevant solution conditions it could be estimated that the cisplatin fraction bound to serum albumin might well exceed $85 \%$ after 24 hours incubation. Though the 1:1 adduct is the most relevant from the physiological point of view, serum albumin was also found to bind cisplatin strongly at higher Pt/HSA stoichiometries even reaching Pt to protein molar ratios around 5/6. Though Cys34 was credited as one of the most likely anchoring sites due to high affinity of platinum to sulfur-containing ligands and given that this target is sterically accessible for drug molecules, no clear proof of direct and preferential platinum coordination to Cys34 was gained. However, some other possible binding sites were also identified. Indeed, Ivanov et al. identified methionine as a primary target by applying an NMR spectroscopy method. Met298 (but not Cys34) was identified as the major sulfur-containing binding site involved in cisplatin interaction with different types of albumin [11]. The major cisplatin binding site appears to be an S,N-macrochelate involving Met298 as a ligand. Apart from Met298, Met87 and Met446 were also proposed to be 
involved in the formation of monofunctional adducts with cisplatin since these residues appear to be solvent exposed [11].

In any case the stability and reversibility of cisplatin binding to serum albumin remained controversial so that the significance of serum albumin binding with respect to the overall pharmacological actions of cisplatin could not be elucidated. The crucial point is to elucidate whether protein binding represents an inactivation process or not.

Here, we will consider the most important advancements of research on the cisplatin serum albumin system registered during the last 15 years. In particular we will concentrate our report on the following issues: 1. The eventual determination of the crystal structure of a cisplatin/serum albumin derivative; 2. The use of ESI-MS as a direct tool to monitor platination of serum albumin; 3. The use of advanced metallomics approaches to describe platination of blood proteins; 4. The detailed evaluation of the pharmacological effects of cisplatin/serum albumin adducts.

\section{The crystal structure of a HSA/Cisplatin adduct}

In 2015 Ferraro et al. solved, for the first time, the crystal structure of a cisplatin/HSA derivative identifyng unambiguously the binding sites for cisplatin [13]. Specifically, this derivative showed at least five distinct platinum binding sites with a quite large degree of Pt occupancy.

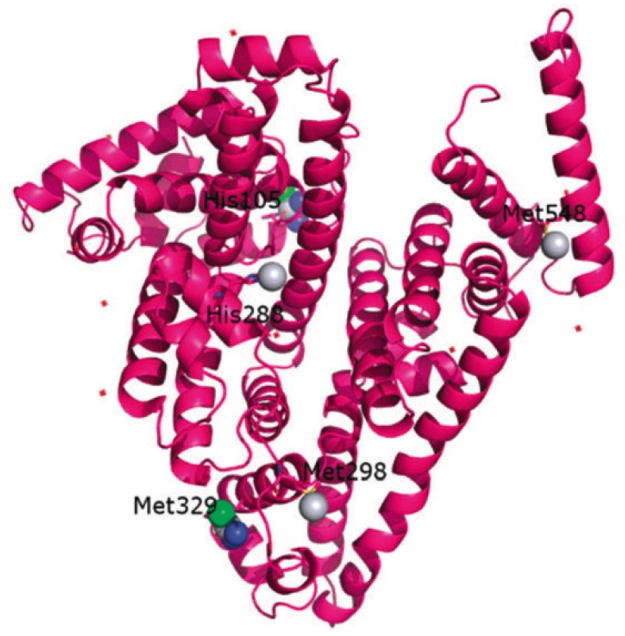

Figure 2. Crystal structure of Human Serum Albumin (from ref. 14).

Crystallographic results point out that cisplatin manifests a clear coordination preference for His and Met side chain residues located on the protein surface. In particular, the drug binds His 105 (subdomain IA), Met298 (domain II, in the loop traversing the two subdomains to link them together), Met329 (subdomain IIB) and Met548 (subdomain IIIB) and the His288 (subdomain IIa). Apparently, His 105 and Met329 seem to be the main binding sites, whereas the others are secondary binding sites, 
as it may be judged from their respective occupancy factors: $\sim 0.8$ for the Pt atoms close to His 105 and Met329 and in the range of $0.30-0.55$ for Pt atoms in other binding sites.

All cisplatin binding sites are located on the protein surface (they do not overlap with fatty acid binding sites) and $\mathrm{Pt}$ is directly coordinated to donor atoms from the protein. The anchoring of the cisplatin fragments (most probably $\left[\mathrm{Pt}\left(\mathrm{NH}_{3}\right)_{2} \mathrm{Cl}\right]^{+}$in line with the literature data) to HSA does not affect the overall protein conformation, although it is associated with a disordering of some protein portions, particularly in domain III.

A

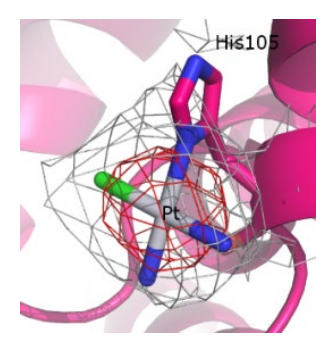

$\mathbf{C}$

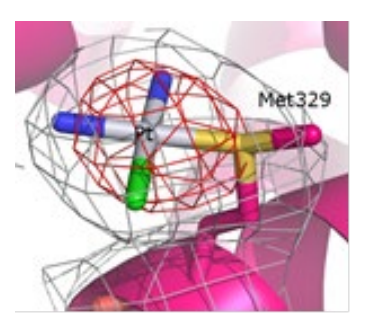

$\mathbf{E}$

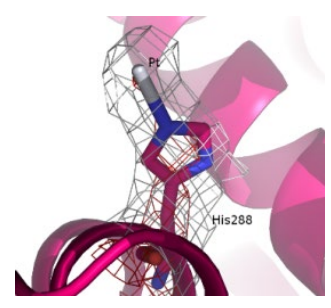

B

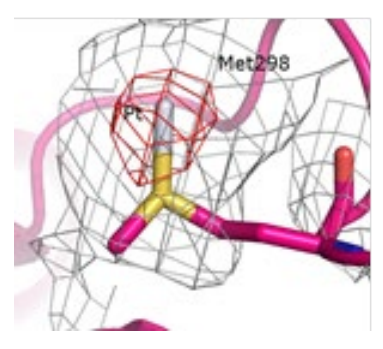

D

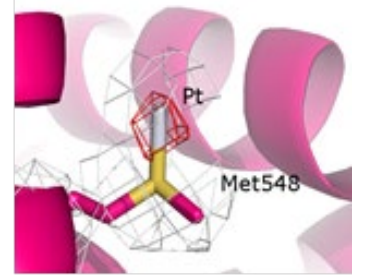

Figure 3. Details of the binding sites of cisplatin on HSA structure in the adduct. The Pt ion is coordinated to His105 (A), Met298 (B), Met329 (C), Met548 (D) and His288 (E). 2Fo-Fc electron density maps are contoured $1.0 \sigma$ (grey) level. The same map has been also contoured at $5 \sigma$ in panel $\mathrm{A}, \mathrm{B}$ and $\mathrm{D}$ (red) and at $2.5 \sigma$ in panel $\mathrm{D}$ and $\mathrm{E}$ (from ref. 14).

These crystallographic results are in good agreement with a few recent studies obtained with other techniques. For instance, based on a mass spectrometry approach, Moraleja et al. identified most of the binding sites located at the HSA surface accessible to cisplatin (five histidines: His9, His67, His105, His128, and H247; two methionines: Met298 and Met329; as well as the N-terminal from the residue Asp13) [14]. These results are largely consistent with crystallographic results. However, 
the crystal structure does not solve all the literature controversies. Discrepancies between the cisplatin binding sites that have been identified by Ferraro et al. and previous results are likely a consequence of the different experimental conditions. Anyway, although solved at a relatively low resolution, the crystal structure of cisplatin/HSA may serve as an excellent starting point at the molecular level to interpret the results of several independent physicochemical experiments carried out so far on this system, as well as theoretical investigations and predictions of cisplatin binding to HSA.

\section{Mass spectrometry as an highly informative tool to investigate cisplatin/HSA interactions}

In recent years, electrospray mass sprectrometry (ESI-MS) has established itself as a potent tool to investigate the ligand-protein interactions at the molecular level. This is particularly true in the case of the interactions of proteins with metal based drugs owing to the frequent formation of tight coordinative bonds. Gibson's group, twenty years ago, first reported a couple of pioneering studies on the ubiquitin-cisplatin system using ESI-MS and demonstrated unambiguously the protein binding of a platinum containing fragment $[15,16]$. Subsequently, the binding site of cisplatin on transferrin was determined by liquid chromatography tandem mass spectrometry (LC-MS/MS), providing the basis for molecular modeling of the transferrin-cisplatin interactions [17]. More recently, Dyson and his coworkers reported a rapid top-down approach to determine the binding sites of Pt-metallodrugs on ubiquitin [18].

From the increasing number of this type of publications, it clearly emerges that mass spectrometry has proven to be a powerful technique for the study of protein/Pt-metallodrug interactions; notably, owing to the latest technological advancements, MS offers today a straightforward and excellent tool to analyze the reactions of metal-based drugs with proteins as large as serum albumin [19]. Moreover, in the very last years, an useful MS computational tool named "Analysis of Protein Modification from Mass Spectra" $\left(\mathrm{Apm}^{2} \mathrm{~s}\right)$ was reported for the identification of the platinum's binding site on a given protein. [20]. This method can be applied more in general to any metal compound endowed with a characteristic isotopic pattern.

Since HSA can bind up to 10 cisplatin moles per mol of protein, it was soon evident that the high stoichiometries of the resulting CDDP-HSA adducts observed could not be explained by the only free cysteine group present in the protein (Cys34). Thus, it was demonstrated that the cleavage of disulphide bonds occurred as a result of the interaction with cisplatin [21]. This was probably followed by an intramolecular interaction and the consequent modification of the secondary structure and the biological functions of the protein. However, even considering Cys 34 and the likely disulfide bonds cleavage, additional binding sites must be involved. Indeed, methionines, histidines or even 
tyrosines have also been suggested as reactive groups towards cisplatin [22]. In that way, a study based on multidimensional protein identification methodology described precisely some binding sites for the Cisplatin-HSA interaction. Sheldrick et al. developed a multidimensional liquid chromatography and ESI tandem mass spectrometry (MudPIT) shotgun proteomics approach to identify the cisplatin binding sites in human serum proteins. A total of five specific binding sites were identified for HSA, including the Cys34, two methionine sites (Met329 and Met548), the tyrosine and aspartate $O$-donor sites Tyr150 and Asp375 [23].

Since almost the totality of the MS methods for the metal/protein's binding site identification require a bottom-up metalloproteomics approach, the stability of metal-protein coordination bonds along the whole process may represent a critical issue. This aspect was extensively analysed in our research group leading to build up a general protocol to test metallodrug-protein adduct stability under the typical conditions of the filter-aided sample preparation (FASP)/bottom-up procedure, ranging from the analysis of solutions containing metal-protein adducts to tandem mass spectrometry experiments [24]. The stability of the metal-protein coordination bonds was addressed also in another pivotal experimental work reported by Gomez-Gomez [14]. In this study a shotgun approach comprising FASP tryptic digestion in combination with peptide-based OFFGEL-IEF and nLC-ESI-LTQ Orbitrap-MS/MS turned out to be an attractive alternative to other previous protein separation-based approaches for the analysis of platinum-protein complexes. The proposed methodology maintains the integrity of platinum complexes throughout all the procedure and makes the identification of platinum-protein derivatives possible even in complex biological samples. Through the inspection of the MS/MS spectra produced with the proposed methodology, these authors characterized most of the binding sites located at the HSA surface accessible to cisplatin. In particular, five histidine residues (His9, His67, His105, His128, and His247) and two methionines (Met298 and Met329), as well as the N-terminal from the residue Asp13 were indicated as preferential platinum's binding sites. The sites located in this work were in agreement with the surface accessibilities of cisplatin in HSA which were obtained by a web-based bioinformatics tool. This work highlighted, once again, the considerable affinity of platinum(II) for the imidazole nitrogens of histidine or the thioether sulphur in methionines. Furthermore, the authors remarked that the proposed methodology also revealed that cisplatin can cross-link residues His67 of domain I and His247 of domain II in HSA.

The majority of the mass spectrometric techniques discussed for the identification of the platinum binding sites involve the preliminary tryptic digestion of the protein followed by the subsequent MS analysis of the resulting peptide mixtures. Nonetheless, some essential information about the binding stoichiometry and the chemical nature of the metal-containing fragments bonded to the proteins can be achieved by so called "native MS". More in general, noncovalent protein-protein and protein- 
ligand complexes interactions relevant for structural biology, can be analyzed by MS thanks to the technological evolution of the available instrumentation [25]. For these types of analyses, assemblies need to be retained in their native quaternary state in the gas phase. This initial small niche of biomolecular mass spectrometry, nowadays often referred to as "native MS," has come to maturation over the last two decades, with dozens of laboratories using it to study mostly protein assemblies. This strategy was also successfully applied in our lab for the study of the protein metalation process using the ESI-Q-Tof high-resolution mass spectrometry.

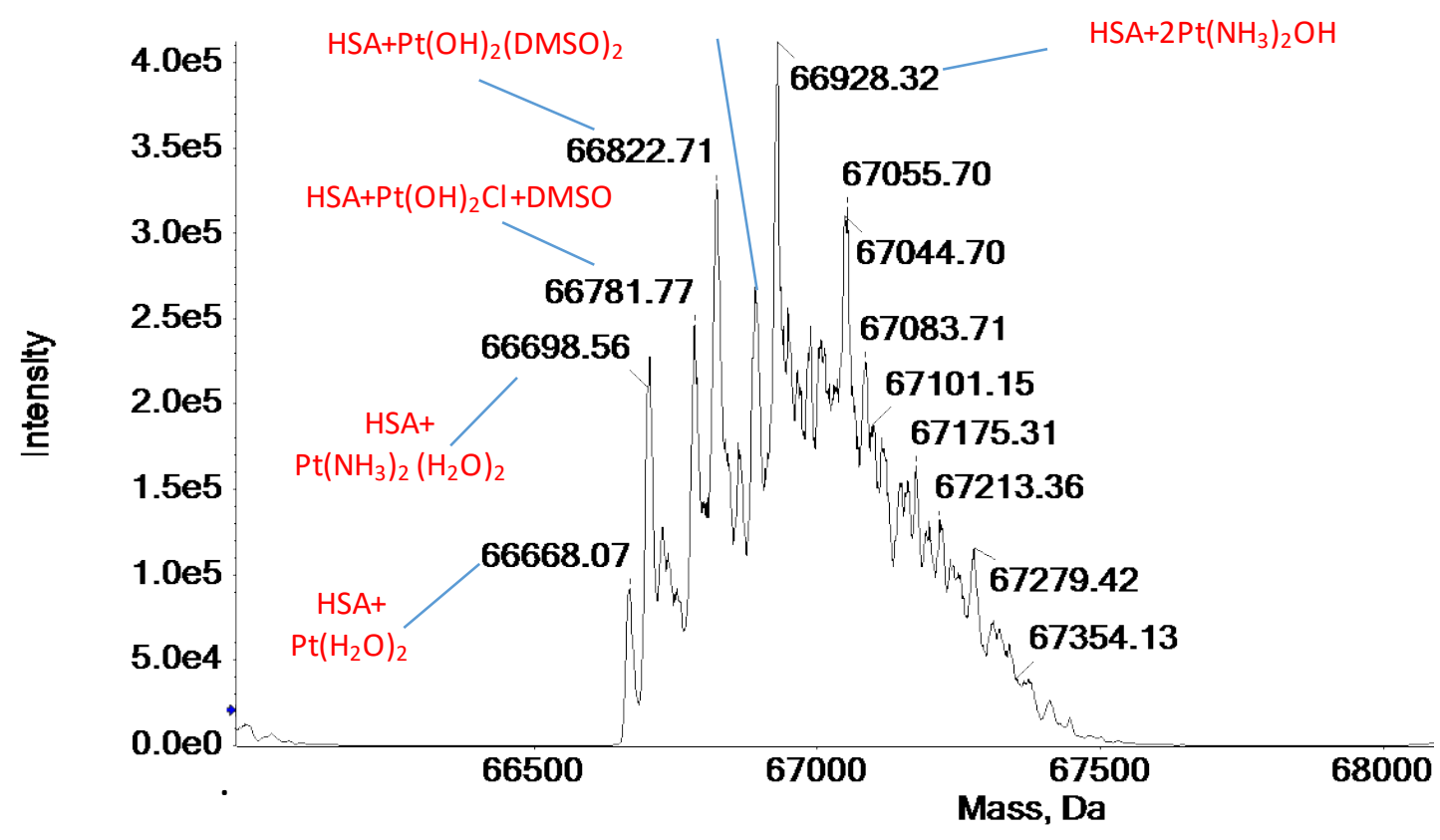

Figure 4. Deconvoluted ESI mass spectrum for CDDP incubated for $3 \mathrm{~h}$ with human serum albumin $10^{-5} \mathrm{M} \mathrm{(3:1} \mathrm{metal} \mathrm{to} \mathrm{protein} \mathrm{molar} \mathrm{ratio)} \mathrm{at} 37^{\circ} \mathrm{C}$ in $20 \mathrm{mM}$ ammonium acetate buffer, $\mathrm{pH} 6.8$.

Specifically, figure 4 shows the ESI-MS spectrum recorded for the platinated HSA in its native conformation. During the incubation with CDDP and the subsequent mass analysis all the physiological-like conditions were maintained in order to preserve the native protein conformation, and then, the right exposition of the binding sites. The results were in perfect agreement with the literature data previously described. In fact, the binding stoichiometry was greater than that permitted by the involvement of Cys34, suggesting a more extensive engagement of other different binding sites. Interestingly, the spectrum showed also a plethora of platinum-containing fragments, some of these retained the ammonia ligands and some other were hydrated, that perfectly fit with the occurrence of CDDP aquation equilibria. Anyway, the eager reactivity of HSA toward Pt was 
evidenced once again in this spectrum: in fact the signal belonging to the unreacted HSA completely disappeared after only $3 \mathrm{~h}$ of incubation.

As the last aspect of the mass spectrometry applied to the study of Cisplatin-HSA interactions, it is worthy to note that few attempts to "fish" the metallated proteins with CDDP have been performed. Steyger and co-workers described a simple and innovative methodology for the identification of CDDP-binding proteins using agarose-conjugated Pt compounds [26]. To identify proteins that bind to cisplatin, they synthesized two different platinum-agarose conjugates, one with two amino groups and another with two chlorides attached to platinum that are available for protein binding, and conducted pull-down assays using cochlear and kidney cells. Mass spectrometric analysis on protein bands after gel electrophoresis identified several proteins, first of all the most abundant HSA, but also myosin IIA, glucose-regulated protein 94 (GRP94), heat shock protein 90 (HSP90), calreticulin, valosin containing protein (VCP), and ribosomal protein L5, as cisplatin-binding proteins.

\section{Application of metallomics to probe the interaction of cisplatin with HSA in blood plasma}

While significant progress has been unquestionably made concerning the identification of the likely molecular mechanism of action of cisplatin [27], a detailed and comprehensive understanding of the biomolecular interactions of Pt-based drugs remains very much a work in progress [28]. This situation must be largely attributed to the relatively limited number of analytical tools that can be used to gain insight into the metabolism of cisplatin in vivo on the one hand and to biological complexity itself on the other. Biochemical transformations that unfold within cells after cisplatin is taken up have received considerable interest [29-31], but the biochemical transformations of Pt-drugs that occur in the bloodstream have received much less attention. This is somewhat surprising given that it has long been known that plasma proteins represent one of the first potential target(s) after the administration of cisplatin $[11,32,33]$ and that from a conceptual point of view all metabolites that are derived from the parent drug in the bloodstream will subsequently interact with cancer cells and healthy tissue cells to determine the intended anticancer effect as well as the unintended off target effects. Accordingly, the direct observation of temporal changes of the Pt speciation in the bloodstream is of crucial importance. To this end, the advent of metallomics tools which allow one to probe the dynamic changes after cisplatin - or any metal-based drug for that matter - is administered into the bloodstream has emerged as a valuable approach in the quest to develop novel metal-based drugs that may offer better selectivity and fewer side effects. While it is logistically challenging to conduct such studies in whole animals, metallomics tools can be conveniently applied to probe the fate of novel metal-based drugs in human plasma in vitro [34], which makes the aforementioned proposition quite attractive 
today. Over the past 15 years a large variety of liquid chromatography-based metallomics approaches have been systematically developed to facilitate the analysis of complex biological fluids for endogenous metalloproteins [35-37]. Unsurprisingly, this conceptual bioanalytical approach was eventually employed to gain insight into the comparative biochemical fate of Pt-based anticancer drugs in human blood plasma in vitro[38].

After blood plasma was obtained from healthy males and females, pharmacologically relevant doses of cisplatin and carboplatin were added to plasma aliquots and the latter were then analysed by sizeexclusion chromatography (SEC) coupled on-line to an inductively coupled plasma atomic emission spectrometer (ICP-AES). In this manner the comparative metabolism of cisplatin and carboplatin could be conveniently observed over time. The results that were obtained by SEC-ICP-AES revealed a negligible inter-individual variation between the metabolism of cisplatin in plasma obtained from males and females and a considerably faster hydrolysis of cisplatin compared to carboplatin (Figure 5), which was in accord with the literature [38]. More importantly, the fact that cisplatin-derived hydrolysis products could be detected within 5 min (Figure 5, inset) clearly demonstrates that even in the presence of $105 \mathrm{mM}$ of chloride in human plasma, cisplatin appears to undergo rapid hydrolysis. At the $3 \mathrm{~h}$ time point the fraction of free cisplatin is $\sim 16 \%$ of total $\mathrm{Pt}$, while $\sim 49.0 \%$ of total Pt eluted bound to HSA and $\sim 8.6 \%$ of total Pt eluted in form of hydrolysis products. Overall, these results clearly demonstrate that metallomics tools, such as SEC-ICP-AES can provide valuable new insight into the metabolism of metal-based drugs in complex human plasma. Furthermore, the results from these investigations provide clear evidence that already 5 min after the addition of cisplatin to human plasma a HSA-Pt complex could be detected.

Since the aforementioned studies clearly showed that HSA represents one important target of cisplatin-derived hydrolysis products in human blood plasma [38] and considering that most cancer patients have lower HSA concentrations in their blood plasma (due to the decreased synthesis, increased catabolism or loss of HSA by kidney damage), [39] the above metallomics tool can be applied to investigate the metabolism of cisplatin in plasma from cancer patients. Also, by plasma fortification with exogenous HSA it is possible to investigate if this may allow one to 'tune' the metabolism of cisplatin [40]. After addition of the same cisplatin dose to plasma aliquots, the concentration of all cisplatin metabolites was measured after 2 hours of incubation. While the analysis of all plasma samples revealed the presence of cisplatin, two hydrolysis products and three platinated plasma proteins, the analysis of plasma from cancer patients revealed $9.8 \%$ less protein bound $\mathrm{Pt}$ (including HSA-Pt complexes) and a 5.3\% increase of the total Pt that eluted in form of hydrolysis products compared to the analysis of plasma from then healthy controls. Since $\left[\mathrm{PtClOH}_{2}\left(\mathrm{NH}_{3}\right)_{2}\right]^{+}$is 
likely the first hydrolysis product that is formed in blood plasma which is also 8-times as toxic as cisplatin $[41,42]$, the rapid formation of this Pt-species might be predominantly responsible for the severe side effects of cisplatin [11,32]. Thus, it was investigated whether cancer patient plasma $(26 \pm 7$ $\mathrm{g} / \mathrm{L}$ of HSA) could be amended with pure exogenous HSA (Alburex) to modulate the metabolism of cisplatin in a desirable way. While cancer patient plasma with HSA concentrations of 36 and $42 \mathrm{~g} / \mathrm{L}$ progressively decreased the concentration of both hydrolysis products at the $2 \mathrm{~h}$ time point, a significant fraction of both hydrolysis products $(12.9 \%$ of total $\mathrm{Pt})$ remained. These in vitro results indicate that boosting the blood plasma HSA concentration of cancer patients with exogenous HSA may reduce the concentration of those species derived by CDDP and reputed highly toxic for the host e.g. $\left[\mathrm{PtClOH}_{2}\left(\mathrm{NH}_{3}\right)_{2}\right]^{+}$. The fact that a significant fraction of both hydrolysis products remains even after boosting the HSA concentration implies that it might be better to 'neutralize' the highly toxic hydrolysis product with a so called 'chemoprotective agent'. To this end, investigation with the metallomics tool have been carried out to demonstrate that sodium thiosulfate [43], $N$-acetylcysteine [44], D-methionine [45], and L-glutathione [46] can modulate the metabolism of cispaltin in human plasma in vitro in a desirable manner. Further studies need to be conducted to identify which of these chemoprotective agents can be co-administered with cisplatin to systematically reduce its severe side effects, while maintaining its anticancer efficacy to transform this metal-based drug into a much safer anticancer drug [32, 33]. 


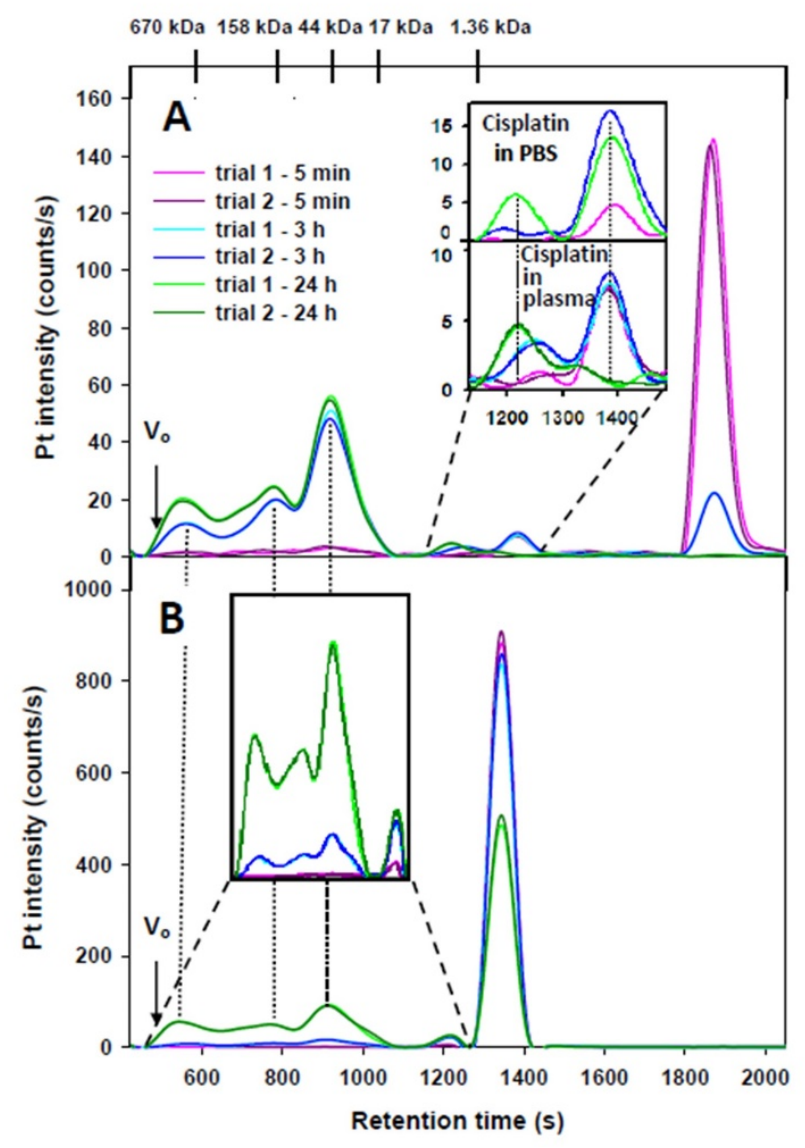

Figure 5. Representative Pt-specific chromatograms (2 replicates superimposed) obtained for the analysis of male human plasma $(3.0 \mathrm{~mL})$ spiked with $(\mathrm{A})$ cisplatin $(0.024 \mathrm{mg} \mathrm{Pt} / \mathrm{mL}$ plasma $)$ and (B) carboplatin $\left(0.12 \mathrm{mg} \mathrm{Pt} / \mathrm{mL}\right.$ plasma). The mixture was incubated at $37^{\circ} \mathrm{C}$ and samples were analysed after $5 \mathrm{~min}$ (pink/purple), $3 \mathrm{~h}$ (light and dark blue) and $24 \mathrm{~h}$ (light and dark green) on a Superdex 200 10/300 GL SEC column ( 30 x $1.0 \mathrm{~cm}$ I.D., $13 \mu \mathrm{m}$ particle size $)$ at $22{ }^{\circ} \mathrm{C}$ using PBS-buffer $(0.15 \mathrm{M}$, pH 7.4) as the mobile phase. Flow rate: $1.0 \mathrm{~mL} \mathrm{~min}^{-1}$, Injection volume: $500 \mu \mathrm{L}$, Detector: ICP-AES at $214.423 \mathrm{~nm}(\mathrm{Pt})$. The retention time of the molecular markers to size calibrate the SEC column are depicted on top. From reference 39. This should be changed to reference 38

\section{Are HSA bound Pt drugs still active?}

The possibility that cisplatin adducts of serum albumin, and more in general adducts of platinum drugs with serum albumin, may retain some degree of biological activity has been long questioned and remains a highly controversial, still unsolved issue. This aspect is very important for its possible pharmaceutical implications. In the case adducts are still active it may be reasonable to prepare a variety of them and test against various cancer models. Indeed, the adducts may cause a different biodistribution of Pt drug and also attenuate the potent systemic toxic effects of free cisplatin; in 
addiction protein cisplatin adducts may serve as a way of selective or preferential drug targeting to tumor tissue. Conversely, if albumin biding represents exclusively a mode of inactivation there is no rationale for this kind of approaches and the binding to albumin should be- at least partially- avoided. This point has now been addressed in two important studies. In $2008 \mathrm{Kratz}$ et al. explored the potential to overcome resistance in cisplatin-resistant A2780 ovarian carcinoma cells by means of macromolecular prodrugs exploiting endocytosis as alternative uptake mechanism [47]. Accordingly, they prepared and investigated two Pt-albumin (PL04-HSA, PL07-HSA) complexes. Intracellular platinum accumulation was quantified by FAAS; Cytotoxic activity was measured using the MTT assay; Endocytosis mechanisms were investigated by co-incubation experiments with bafilomycin A(1). Whereas the intracellular accumulation of the low molecular precursors PL04 and PL07 was reduced in the resistant cell variant, no difference between sensitive and resistant cells was observed for the macromolecular complexes. In the presence of bafilomycin A(1), an inhibitor of clathrinmediated endocytosis, intracellular accumulation of theinvestigated macromolecular complexes was decreased. In conclusion, cellular accumulation of macromolecular platinum complexes turned out not to be altered in cisplatin-resistant A2780 cells as these complexes enter the cells mainly via endocytotic pathways. According to that study, macromolecular platinum complexes specially designed to circumvent reduced cellular accumulation resulted to be a promising approach to overcome cisplatin resistance.

Another remarkable study by Sturup and coworkers appeared in 2010 [48]. They prepared an equimolar human serum albumin-cisplatin adduct (HSA-Pt) and investigated its actions toward either in suspension Ehrlich Ascites Tumor Cells (EATC) or adherent Ehrlich Lettre'Ascites Cells (Lettre') at 10 micromolar concentration. Notably, it was clearly observed that HSA-Pt at variance with free cisplatin did not produce cell death nor was taken up by the cells to any significant extent within 24 $\mathrm{h}$ incubation. The accumulation and cytotoxicity of HSA-Pt was compared to $10 \mathrm{mmol} / \mathrm{L}$ cisplatin for which a far larger accumulation and cytotoxicity were observed in EATC compared to Lettre'. Cisplatin was taken up by both cell lines in appreciable amounts, however, to a notably larger extent in EATC, where it also showed greater cytotoxicity. The stability of the platinum species was also determined using size exclusion chromatography-inductively coupled plasma-mass spectrometry (SEC-ICP-MS). Results point out that albumin bound cisplatin at 1:1 molar ratio seems to be virtually inactive; at the same time these experiments proved that the stability of the investigated compound may be a critical factor in affecting the observed results. Indeed, HSA-Pt was found to be stable in Hanks Balanced Saline Solution (HBSS) and in Phosphate Buffered Saline (PBS) at pH 5.3, 6.1 and 7.4. HSA-Pt was unstable in RPMI-1640 with and without $10 \%$ FCS, while cisplatin was unstable in RPMI-1640 with and without 10\% serum. These experiments show that the stability of the test 
compounds in the cell medium should be preliminarily investigated before performing cell experiments.

Overall, these studies concerning the activity of protein bound platinum species suggest that a variety of scenarios are possible where the cytotoxic activity of the protein bound platinum species may be retained, attenuated or even lost. These results warrant further and more systematic investigations of these issues as results may critically depend on the nature of the protein adduct, the type of Pt protein interaction as well as the type of investigated cancer cells.

\section{Concluding Remarks}

In conclusion, we have shown here that significant progress has been made in the comprehension of the cisplatin/serum albumin system. The most relevant progress was obtained with regard to the molecular characterization of this system. Indeed the crystal structure has been solved at a moderate resolution that permits yet the unambiguous identification of the metalation sites. Five distinct binding sites could be identified as methionine and histidine side-chains. Platinum binding occurs through formation of coordinative bonds and is rather stable. The various sites show comparable levels of occupancy by Pt implying that their binding affinities are not so different. The typical platinum fragment is $\left[\mathrm{Pt}\left(\mathrm{NH}_{3}\right)_{2}\right]^{2+}$. Notably this picture has been largely confirmed by a number of ESI-MS studies. Conversely, metallomics studies offered clear evidence concerning the mode of platination of serum proteins following cisplatin administration. It was observed that cisplatin becomes protein-bound after a relatively short incubation time. Platination involved mostly albumin and to a smaller degree other serum proteins.

Finally, the controversial issue of the residual activity of the cisplatin albumin system was investigated in a couple of studies. Kratz et al. [47] demonstrated that macromolecular complexes of platinum drugs with serum albumin remain cytotoxic and are able to overcome cisplatin resistance. Conversely Sturup et al. [48] by investigating the equimolar 1:1 adduct of cisplatin with serum albumin in only two cell lines observed that the cisplatin serum albumin adduct at variance with free cisplatin is not able to induce Pt cell accumulation nor to cause cancer cell death through apoptosis. This finding might imply that cisplatin binding to serum albumin at least under the investigated experimental conditions and in relation to the studied cell lines mostly represents a mechanism of cisplatin inactivation.

These contractictory results on such a crucial issue warrant wider and more systematic studies on the in vitro anticancer activity of platinum drug albumin conjugates. It seems that the residual activity of 
these species is critically dependent on the metal/protein stoichiometry, the nature of Pt biding to protein side chains and also the precise nature of the tested cancer cell lines.

\section{Acknowledgments}

The authors thank Beneficentia Stiftung (Vaduz, Liechtenstein), AIRC and Fondazione Cassa Risparmio Firenze for founding the projects “Advanced mass spectrometry tools for cancer research: novel applications in proteomics, metabolomics and nanomedicine" (Multi-user Equipment Program 2016, Ref. code 19650). CIRCMSB is also acknowledged. TM thanks University of Pisa (Fondi Ateneo Rating 2018) and Fondazione Italiana per la Ricerca sul Cancro, Project Code: 18044. JG greatly acknowledges funding from CIHR-THRUST and Alberta Innovates Health Solutions (HIS grant 201000689).

\section{References}

[1] A.A. Legin, A. Schintlmeister, M. A. Jakupec, M. Galanski, I. Lichtscheidl, M. Wagner, B. K. Keppler, Chem. Sci. 5 (2014) 31-35.

[2] A.R. Timerbaev, C.G. Hartinger, S.S. Aleksenko, B. K. Keppler, Chem. Rev. 106 (2006) 22242248.

[3] L. Messori, A. Merlino, Coord. Chem. Rev. 315(2016) 67-89

[4] Peters, T. (1996). All About Albumin: Biochemistry, Genetics and Medical Applications. San Diego, CA: Academic Press Limited.

[5] X.M. He, D.C. Carter, Nature 358 (1992) 209-215.

[6] S. Sugio, A. Kashima, S. Mochizuki, M. Noda, K. Kobayashi, Protein Eng. 12 (1999) 439-46.

[7] G. Sudlow, D.J. Birkett, D.N. Wade, Mol. Pharmacol. 12 (1976)1052-61.

[8] U. Kragh-Hansen, Pharmacol. Rev. 33 (1981) 17-53

[9] T.W. Evans Aliment. Pharmacol. Ther.16 (2002) 6-11.

[10] W. Bal, M. Sokołowska, E. Kurowska, P. Faller, Biochim Biophys Acta. 1830 (2013) 54445455. 
[11] A.I. Ivanov, J. Christodoulou, J.A. Parkinson, K.J. Barnham, A. Tucker, J. Woodrow, P.J. Sadler, J. Biol. Chem. 273 (1998) 14721-14730.

[12] B.P. Espósito, R.Najjar, Coord. Chem. Rev. 232 (2002) 137-149.

[13] a) G. Ferraro, L. Massai, L.Messori, A. Merlino, Chem. Commun. 51 (2015) 9436-9439; b) A. Merlino, T. Marzo, L. Messori, Chem. Eur. J. 23 (2017) 6942 - 6947.

[14] I. Moraleja, E. Moreno-Gordaliza, D. Esteban-Fernández, M. L. Mena, M. W. Linscheid, M. M. Gómez-Gómez, Anal. Bioanal. Chem. 407 (2015) 2393-2403.

[15] D. Gibson, C.E. Costello, Eur. Mass Spectrom. 5 (1999) 501-510.

[16] T. Peleg-Shulman, D. Gibson, JACS 123 (2001) 3171-3172.

[17] I. Khalaila, C.S. Allardyce, C.S. Verma, P.J. Dyson, Chembiochem: Eur. J. Chem. Biol. 6 (2005), 1788-1795.

[18] C.G. Hartinger, Y. Tsybin, J. Fuchser, P.J. Dyson, Inorg. Chem. 47 (2008) 17-19.

[19] A. Pratesi, D. Cirri, L. Ciofi, L. Messori, Inorg. Chem. 57 (2018) 10507-10510.

[20] R. F. S. Lee, L. Menin, L. Patiny, D. Ortiz, P. J. Dyson, Anal. Chem. 89 (2017) 11985-11989.

[21] A. Sugii, K. Nishimura, K. Harada, M. Nakayama, S. Masuda, Chem. Pharm. Bull. 39 (1991) 408-410.

[22] M. Wenzel, A. Casini, Coord. Chem. Rev. 352 (2017) 432-460.

[23] J. Will, D. A. Wolters, W. S. Sheldrick, ChemMedChem 3 (2008) 1696-1707.

[24] E. Michelucci, G. Pieraccini, G. Moneti, C. Gabbiani, A. Pratesi, L. Messori, Talanta 167 (2017) $30-38$.

[25] A. C. Leney, A. J. R. Heck, J. Am. Soc. Mass Spectrom. 28 (2017) 5-13.

[26] T. Karasawa, M. Sibrian-Vazquez, R. M. Strongin, P. S. Steyger, PlosOne 8 (2013) e66220.

[27] S.E. Sherman, S.J. Lippard, Chem. Rev. 87 (1987) 1153-1181. Please replace this reference with D. Gibson, Dalton Trans. (2009) 10681-10689 (thanks!)

[28] X. Wang, Z. Guo, Anti-Cancer Agents Med. Chem. 7 (2007) 19-34.

[29] A. Maccio, C. Madeddu, Expert Opin. Pharmacother. 14 (2013) 1839-1857. 
[30] S.M. Sancho-Martinez, L. Prieto-Garcia, M. Prieto, J.M. Lopez-Novoa, F.J. Lopez-Hernandez, Pharmacol. \& Therap. 136 (2012) 35-55.

[31] C.-P. Tan, Y.-Y. Lu, L.-N. Ji, Z.-W. Mao, Metallomics 6 (2014), 6, 978-995.

[32] M. Sooriyaarachchi, G.N. George, I.J. Pickering, A. Narendran, J. Gailer, Metallomics 8 (2016) 1170-1176.

[33] J.Gailer, J. Inorg. Biochem. 179 (2018) 154-157.

[34] B.W.J. Harper, T.T. Morris, J. Gailer, J.R. Aldrich-Wright, J. Inorg. Biochem. 163 (2016) 95102.

[35] J.L. Gomez-Ariza, E.Z. Jahromi, M. Gonzalez-Fernandez, T. Garcia-Barrera, J. Gailer, Metallomics 3 (2011) 566-577.

[36] J.P. Barnett, D.J. Scanlan, C.A. Blindauer, Anal. Bioanal. Chem. 402 (2012) 3311-3322.

[37] M.P. Chantada-Vasquez, A. Moreda-Pineiro, M.C. Barciela-Alonso, P. Bermejo-Barrera, Appl. Spectrosc. Rev. 52 (2017) 145-174.

[38] M. Sooriyaarachchi, A. Narendran, J. Gailer, Metallomics 3 (2011), 49-55.

[39] W.Y. Craig, T.B. Ledue, R.F. Ritchie, Plasma proteins. Clinical Utility and Interpretation; Dade Behring Inc.: Newark (2000).

[40] T.T. Morris, Y. Ruan, V.A. Lewis, A. Narendran, J. Gailer, Metallomics 6 (2014) 2034-2041.

[41] P. Videhult, G. Laurell, I. Wallin, H. Ehrsson, Exp. Biol. Med. 231(2006) 1638-1645.

[42] J.H. Van den Berg, J.H. Beijnen, A.J.M. Balm, J.H.M. Schellens, Cancer Treat. Rev. 32 (2006) 390-397.

[43] M. Sooriyaarachchi, A. Narendran, J. Gailer, Metallomics 4 (2012) 960-967.

[44] M. Sooriyaarachchi, A. Narendran, J. Gailer, Metallomics 5 (2013) 197-207.

[45] M. Sooriyaarachchi, A. Narendran, W.H. White, J. Gailer, Metallomics 6 (2014) 532-541.

[46] M. Sooriyaarachchi, M.A. Gibson, B.d.S. Lima, J. Gailer, J., Can. J. Chem. 94 (2016) 360-366.

[47] D. Garmann, A. Warnecke, G.V. Kalayda, F. Kratz, U. Jaehde, J. Control. Release 131 (2008) $100-106$ 
[48] C. Møller, H.S. Tastesen, B. Gammelgaard, I.H. Lambert, S. Stürup, Metallomics 2 (2010) 811818. 